\title{
Fibroma Odontogénico Periférico: Reporte de un Caso
}

\author{
Peripheral Odontogenic Fibroma: Case Report
}

\author{
Francisco Pinedo ${ }^{1,4,5}$; Alejandra Fernández ${ }^{2,3}$; Cristian Bravo'; Carolina Somarriva1 \& Natalia Santibañez ${ }^{1}$
}

PINEDO, F.; FERNÁNDEZ, A.; BRAVO, C.; SOMARRIVA, C. \& SANTIBAÑEZ, N. Fibroma odontogénico periférico: reporte de un caso. Int. J. Odontostomat., 11(2):178-181, 2017.

RESUMEN: El Fibroma Odontogénico Periférico fue definido por la OMS en el año 2005 como una neoplasia benigna rara constituida por tejido fibroso maduro y una cantidad variable de epitelio odontogénico inactivo. Dada su presentación clínica, localización y baja prevalencia suele ser mal diagnosticado como una lesión reaccional. Se presenta un caso clínico de fibroma odontogénico periférico tratado mediante remoción quirúrgica y se realiza una revisión de la bibliografía respecto a la patología con el propósito de esclarecer algunos aspectos de esta lesión, además de incluirla dentro de los posibles diagnósticos diferenciales de lesiones reaccionales gingivales. El objetivo del siguiente artículo es presentar un caso clínico de FOP tratado mediante remoción quirúrgica y aportar en el diagnóstico diferencial de las lesiones reaccionales gingivales.

PALABRAS CLAVE: tumores odontogénicos, neoplasias gingivales, fibroma odontogénico periférico, tumoraciones gingivales.

\section{INTRODUCCIÓN}

El Fibroma odontogénico periférico (FOP) fue descrito por primera vez por Gardner, en 1980, como la contraparte extraósea del fibroma odontogénico central y en el año 1982 lo diferenció del fibroma osificante periférico (Gardner, 1982). Luego, de Villiers Slabbert \& Altini (1991) agregaron que es una neoplasia fibroblástica odontogénica. Actualmente, la OMS lo define como una neoplasia benigna rara constituida por tejido fibroso maduro, con variada cantidad de epitelio odontogénico inactivo (Barnes et al., 2005).

Los aspectos epidemiológicos descritos en la literatura con respecto al FOP son controvertidos. Daley \& Wysocki en el año 1994 reportaron que el FOP correspondía al tercer tumor odontogénico mas frecuente. Ritwik \& Brannon (2010) determinaron que correspondía al $23 \%$ del total de tumores odontogénicos registrados. Con respecto a la predilección por sexo, por un lado se considera que no existe (Alaeddini et al., 2010) y por otro lado, se considera que las mujeres se ven más afectadas (Lin et al., 2008). En relación a la localización tampoco existe un acuerdo, se ha descrito que presenta una mayor prevalencia en la mandíbula, en el maxilar (Lin et al.) o sin predilección (Manor et al., 2004; Alaeddini et al.). Pero si está claro que la encía adherida y usualmente en la zona premolar y molar, ya sea de maxilar o mandíbula es la más comprometida (Manor et al.). Generalmente el FOP se desarrolla entre la segunda y cuarta década (Manor et al.; Lin et al., 2008; Alaeddini et al.).

En relación a los aspectos clínicos de FOP, generalmente se describe como una masa gingival de crecimiento lento que puede aparecer entre los dientes y causar el desplazamiento de estos (Shafer et al., 2006). También se señala que se presenta como una tumoración exofítica, de crecimiento lento, sésil o pedunculado, recubierto por mucosa de coloración normal a la adyacente y asintomática (Ritwik \& Brannon).

\footnotetext{
${ }_{1}^{1}$ Facultad de Odontología, Universidad Andrés Bello, Chile.

2 DDS, MSC, Profesor Asistente, Facultad de Odontología Universidad Andrés Bello, Chile.

${ }^{3}$ Centro de Investigación Biomédica, Universidad de Los Andes, Chile.

${ }^{4}$ Cirujano Maxilofacial Hospital Santiago Oriente, Dr. Luis Tisné Brousse, Chile.

${ }^{5}$ Cirujano Maxilofacial Hospital Dr. Luis Calvo Mackenna, Santiago, Chile.
} 
Se describen dos variables histopatológicas dependiendo de la cantidad de restos epiteliales odontogénicos que posea. Su variante hipocelular, se dispone sobre un tejido fibromixoide, no infiltrante, que puede formar calcificaciones y que recuerda a un foliculo dental. La variante rica en células epiteliales, se dispone sobre una cantidad variable de colágeno, con zonas hipocelulares y áreas vasculares. En este segundo tipo de FOP, se puede formar tejido calcificado de carácter metaplásico, que recuerda al cemento, hueso osteoide o dentina. En ambos tipos histologicos es rara la presencia de una capsula bien definida (Barnes et al.).

\section{REPORTE DE CASO}

Paciente de sexo femenino de 46 años de edad fue derivada al Servicio de Cirugía Oral y Maxilofacial de la Facultad de Odontología de la Universidad Andrés Bello. Consultó por aumento de volumen en el maxilar, con un año de evolución y asintomático. La paciente es diabética no controlada, hipertensa y padece de Síndrome de Turner.

Al examen intraoral se observó una tumoración ovoídea de consistencia firme, base pediculada amplia, de aproximadamente dos centímetros de diámetro en su eje mayor. Su superficie se encuentra parcialmente erosionada con áreas pálidas. La lesión se encuentra asociada a la encía libre y adherida de la zona bucal de canino y premolares de la hemimaxila izquierda, extendiéndose al área palatina. El segundo premolar izquierdo se encontraba en estado de raíz (Fig. 1). Se corroboró que no hubiese lesión ósea asociada al diente. (Fig. 3).

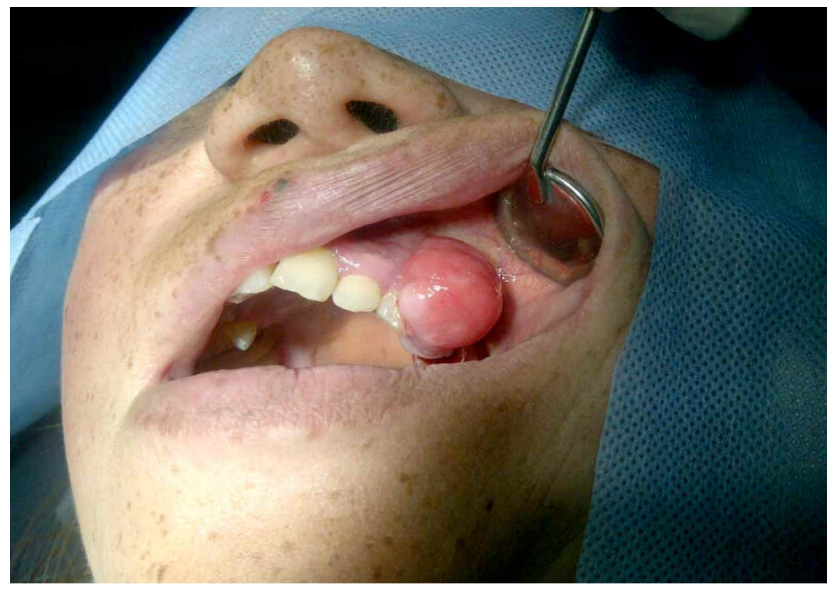

Fig. 1. Tumoración ovoidea en relación a zona de canino y premolares izquierdos en la maxila.
Debido a las características clínicas, ubicación y prevalencia, la hipótesis diagnóstica formulada fue de granuloma piogénico.

Como tratamiento se realizó una biopsia excisional de la tumoración, abarcando un área de 5 $\mathrm{mm}$ alrededor de la base del pedículo, incluyendo el periostio subyacente.

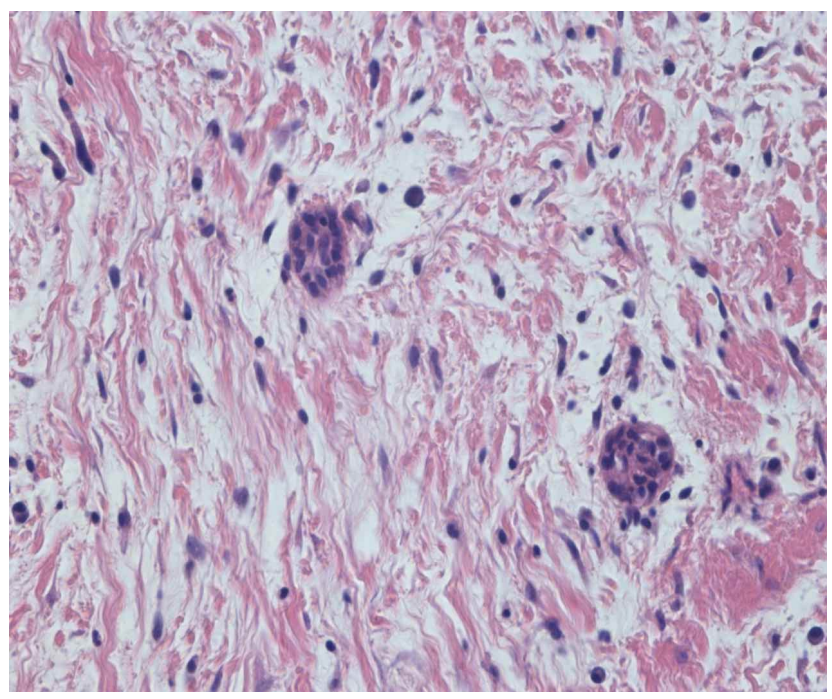

Fig. 2. Muestra histopatológica, tinción hematoxilina eosina, 40X. Servicio de histopatología Universidad Andrés Bello. Proliferación fibrocelular de células fusadas con gran componente colágeno. Se observan remanentes epiteliales en forma de nidos.

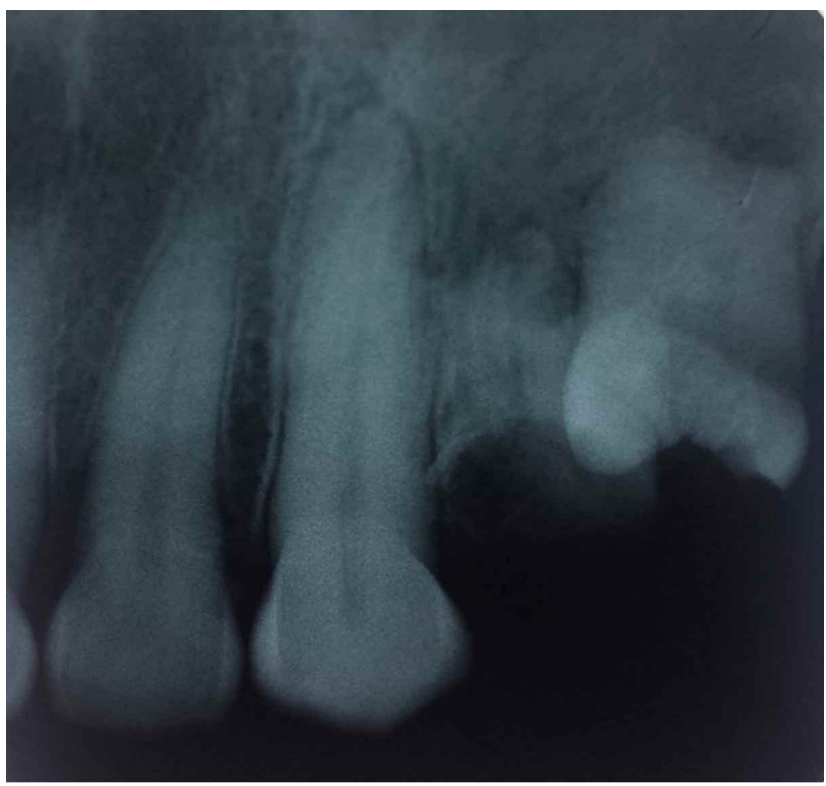

Fig. 3. Radiografía retroalveolar de zona afectada.Se observa el diente 25 al estado de raíz. 
Al examen histopatológico se observó una masa tumoral constituida por una proliferación fibrocelular, de células fusadas, con gran componente colágeno dispuesto en todos los sentidos del espacio. Incluye remanentes epiteliales en forma de nidos y cordones celulares y una que otra calcificación irregular. En toda la muestra se observa variable grado de infiltrado inflamatorio, siendo más intenso hacia la periferia, donde aparece el epitelio de revestimiento parcialmente ulcerado (Fig. 2).

Se controló una semana posterior al tratamiento quirúrgico donde la mucosa comprometida se observó normal.

\section{DISCUSIÓN}

El Fibroma Odontogénico Periférico es una neoplasia benigna odontogénica rara, de baja prevalencia e incidencia y entre los diferentes tumores odontogénicos periféricos es el más frecuente (Manor et al.).

Debido a su presentación clínica, ubicación y escasa frecuencia, es que la mayoría de las veces es diagnosticada inicialmente como una lesión reaccional. Se describe que entre sus diagnósticos diferenciales se debe incluir granuloma piogénico, hiperplasia fibrosa epitelial inflamatoria, y fibroma osificante periférico (Baiju \& Rohatgi, 2011). El examen radiográfico permite descartar un origen central con consecuente exteriorización.

Histológicamente es importante diferenciar estas lesiones. El Granuloma Piogénico muestra una proliferación vascular similar a la del tejido de granulación, presenta numerosos vasos sanguíneos llenos de eritrocitos, un infiltrado inflamatorio mixto constituido por linfocitos, neutrófilos y plasmocitos, además su superficie usualmente se encuentra ulcerada. La presencia de epitelio odontogénico, tejido conectivo fibromixomatoso y ocasionalmente sitios de calcificación (dentinoide, cementoide o osteoide), que son hallazgos vistos en el Fibroma Odontogénico Periférico, no se ven en el Granuloma Piogénico (Sreeja et al., 2014).

El Fibroma Osificante Periférico se caracteriza por regiones calcificadas que se evidencian radiográficamente (Neville et al., 2009). Histológicamente, es común observar proliferación fibroblástica en conjunto con focos de mineralización con variados grados de calcificación, presentando hueso, cementoide o calcificaciones distróficas. La presencia de epitelio odontogénico, vista solo en el Fibroma Odontógenico Periférico es un hallazgo que lo diferencia del Fibroma Osificante Periférico (Sreeja et al.).

En este caso se diagnosticó inicialmente como granuloma piogénico asociándolo a la presencia del premolar en estado de raíz y debido a su alta frecuencia, al aspecto clínico y localización. Aunque se haya errado en el diagnóstico presuntivo de Granuloma Piogénico, no se alteró el plan de tratamiento propuesto, debido a que este es el mismo para todas las lesiones nombradas (Silva et al., 2012).

El procedimiento indicado consiste en una biopsia excisional de la lesión (Silva et al.). Se ha reportado, que la recurrencia del FOP se ha asociado con presencia histológica de actividad en la capa basal del epitelio. Por otro lado, baja recurrencia se ha asociado con la presencia de calcificaciones en oposición a los restos de epitelio odontogénico (Ritwik \& Brannon). Sin embargo, otros autores señalan la escasa evidencia que existe en relación al pronóstico y a la probabilidad de recurrencia, por lo que se recomienda su seguimiento y control periódico (Alaeddini et al.; Silva et al.).

PINEDO, F.; FERNÁNDEZ, A.; BRAVO, C.; SOMARRIVA, C. \& SANTIBAÑEZ, N. Peripheral odontogenic fibroma: case report. Int. J. Odontostomat., 11(2):172-181, 2017.

ABSTRACT: Peripheral odontogenic fibroma was described by the World Health Organization (WHO) in 2005, as a rare benign tumor containing mature fibrous connective tissue with a varying amount of inactive odontogenic epithelium. Though its clinical presentation, localization and low prevalence, it tends to be misdiagnosed as a reactive lesion. We present a case report of a Peripheral Odontogenic Fibroma treated by surgical resection and a narrative review of the literature with the purpose of clarifying different aspects of this lesion besides considering it as a possible differential diagnosis of reactive gingival lesions. The purpose of this article is to present a case report of peripheral odontogenic fibroma treated by surgical resection. Also to contribute to the differential diagnosis of gingival reactive lesions.

KEY WORDS: odontogenic tumors, gingival neoplasms, peripheral odontogenic fibroma, gingival swellings. 


\section{REFERENCIAS BIBLIOGRÁFICAS}

Alaeddini, M.; Salehizadeh, S.; Baghaii, F. \& Etemad-Moghadam, $\mathrm{S}$. A retrospective analysis of peripheral odontogenic fibroma in an Iranian population. J. Oral Maxillofac. Surg., 68(9):2099-103, 2010.

Baiju, C. S. \& Rohatgi, S. Peripheral odontogenic fibroma: A case report and review. J. Indian Soc. Periodontol.,15(3):273-5, 2011.

Barnes, L.; Eveson, J. W.; Reichart, P. \& Sidransky, D. (Eds.). World Health Organization Classification of Tumours. Pathology and Genetics of Head And Neck Tumours. Lyon, IARC Press, 2005.

Daley, T. D. \& Wysocki, G. P. Peripheral odontogenic fibroma. Oral Surg. Oral Med. Oral Pathol., 78(3):329-36, 1994

de Villiers Slabbert, H. \& Altini, M. Peripheral odontogenic fibroma: a clinicopathologic study. Oral Surg. Oral Med. Oral Pathol., 72(1):86-90, 1991.

Gardner, D. G. The peripheral odontogenic fibroma: an attempt at clarification. Oral Surg. Oral Med. Oral Pathol., 54(1):40-8, 1982.

Lin, C. T.; Chuang, F. H.; Chen, J. H.; Chen, C. M. \& Chen, Y. K. Peripheral odontogenic fibroma in a Taiwan chinese population: a retrospective analysis. Kaohsiung J. Med. Sci., 24(8):415-21, 2008.

Manor, Y.; Mardinger, O.; Katz, J.; Taicher, S. \& Hirshberg, A. Peripheral odontogenic tumours--differential diagnosis in gingival lesions. Int. J. Oral Maxillofac. Surg., 33(3):268-73, 2004.

Neville, B. W.; Damm, D. D.; Allen, C .M. \& Bouquot, J. E. Oral and Maxillofacial Pathology. $3^{\text {rd }}$ ed. Philadelphia, W.B. Saunders, 2009.

Ritwik, P. \& Brannon, R. Peripheral odontogenic fibroma: a clinicopathologic study of 151 cases and review of the literature with special emphasis on recurrence. Oral Surg. Oral Med. Oral Pathol. Oral Radiol Endod., 110(3):357-63, 2010.

Shafer, W. G.; Hine, M. K. \& Levy, B. M. Textbook of Oral Pathology. $5^{\text {th }}$ ed. New Delhi, Elsevier, 2006.

Silva, B. S. F.; Yamamoto, F. P.; Da Costa, R. M. B.; Silva, B. T. C.; Carvalho, W. R. S. \& Pontes, H. A. R. Peripheral odontogenic fibroma: Case report of a rare tumor mimicking a gingival reactive lesion. Rev. Odontol. U. N. E. S. P., 41(1):64-7, 2012.

Sreeja, C.; Vezhavendan, N.; Shabana, F.; Vijayalakshmi, D.; Devi, M. \& Arunakiry, N. Recurrent peripheral odontogenic fibroma associated with basal cell budding. J. Pharm. Bioallied. Sci., 6(1):S204-7, 2014
Dirección para correspondencia:

Alejandra Fernández

Facultad de Odontología

Universidad Andres Bello

Echaurren 237

Santiago

CHILE

E-mail: Alejandra.fernandez@unab.cl

Recibido : 22-11-2016

Aceptado: 04-04-2017 